\title{
IgA NEPHROPATHY PRESENTING AS RAPIDLY PROGRESSIVE GLOMERULONEPHRITIS- A CASE REPORT
}

\author{
Gopal Prasad ${ }^{1}$, Kumar Abhinav²
}

${ }_{1}^{1}$ Associate Professor, Department of Nephrology, Patna Medical College, Patna, Bihar, India.

2Junior Resident, Department of Internal Medicine, Patna Medical College, Patna, Bihar, India.

HOW TO CITE THIS ARTICLE: Prasad G, Abhinav K. IGA nephropathy presenting as rapidly progressive glomerulonephritis- a case report. J. Evolution Med. Dent. Sci. 2019;8(03):227-229, DOI: 10.14260/jemds/2019/49

\section{PRESENTATION OF CASE}

45-year-old female patient a house wife from a rural area of Samastipur district was admitted at Patna medical college on 10 august 2015 with complaints of -

Anorexia, nausea, vomiting, mild swelling of both feet for 3 weeks.

No comorbidities like jaundice, arthralgia/arthritis, rash, COPD or occupational exposure to agricultural wastes, no gynaecological problem.

No history of hypertension and diabetes. No recent history of impetigo, sore throat including tonsillitis. No addiction and drug abuse.

At the time of admission, she had mild anaemia, was febrile (100.6 F), respiratory rate $24 / \mathrm{min}$, icterus-absent, pedal oedema $1+$, no sacral oedema, pulse $80 /$ min regular, no radiofemoral or radio-radial delay, supine B.P. in rt. arm; 130/90 (on CCB, BB started from outside private clinic 1 week), periorbital oedema.

No positive finding in systemic examination.

Urinalysis showed 3+ proteinuria, haematuria (30-40/ hpf), pyuria (30-40/hpf) but no cast. Severe renal failure (Serum creatinine 5.7). She was advised for dialysis (intermittent HD through temporary internal jugular catheter) and renal biopsy was planned in view of no obvious infective aetiology and rapid decline of renal function and glomerular proteinuria within last 2-3 week.

During hospital stay after 1 week she became anuric, developed accelerated hypertension and LVF and respiratory distress. She was managed with more frequent HD (4 per week) with ultrafiltration and increased dose of antihypertensive and diuretics. Broad spectrum antibiotics and antifungal were started to which she responded favourably. After resolution of pneumonia and BP control renal biopsy was performed. In the meantime, serology wasANA, C-ANCA, P-ANCA, ANTI GBM titer all within normal limit.

Post biopsy was uneventful. Patient remained anuric during entire hospital stay.

\section{CLINICAL DIAGNOSIS}

RPGN, Idiopathic.

'Financial or Other Competing Interest': None.

Submission 22-10-2018, Peer Review 28-11-2018,

Acceptance 04-12-2018, Published 21-01-2019.

Corresponding Author:

Dr. Gopal Prasad,

\#201, Shristi Enclave,

Opposite Kankarbagh PS,

Kankarbagh,

Patna-800020,

Bihar, India.

E-mail: drgopal.prsd@gmail.com

DOI: $10.14260 /$ jemds/2019/49

(c) $(i)(9)$

\section{DIFFERENTIAL DIAGNOSIS}

1. RPRF, ATIN.

2. RPGN, Renal Limited Vasculitis, ANCA-Negative.

3. Pulmonary-Renal Syndrome, Anti-GBM Antibody Mediated Disease.

\section{PATHOLOGICAL DISCUSSION}

Urinalysis showed 3+ proteinuria, haematuria (30-40/ hpf), pyuria (30-40/hpf) but no cast. Severe renal failure (Serum creatinine 5.7).

Renal biopsy sample had 26 glomeruli, 14 (53.8\%) were globally sclerosed. Of the remaining glomeruli, 10 (38.4\%) exhibit crescents- 5 were cellular, 2 fibro-cellular and 3 fibrous crescents. In IF there were $3+$ mesangial IgA deposits and negative for IgM, C-3 and C1q. Interstitium was oedematous with diffuse inflammatory cell infiltration both mononuclear neutrophil and eosinophil. Tubular atrophy and interstitial fibrosis involved $<10 \%$ of the sample cortex. 3 glomeruli display segmental fibrinoid tuft necrosis.

\section{DISCUSSION OF MANAGEMENT}

Patient remained admitted for 1 month. She remained anuric throughout and dialysis dependent. She was treated with I.V. then oral steroids and I.V. Cyclophosphamide for focal necrotizing IgA nephropathy with crescents.[1,2] At the end of 1-month she was discharged in a stable condition and was counseled for need of long term RRT. She continued thrice a week HD as OPD patient. She remained anuric for last 2 month. So, immunosuppression was stopped for expected poor response in this case.

At the end of 2 nd month she developed high grade fever and relapse of pneumonic consolidation in $x$ ray. She was again managed with antimalarial, antibiotic and nebulization, antifungal. She succumbed in ICU due to massive pneumonia and severe sepsis.

IgAN is the most common primary GN in the world.(3) The prevalence rate varies across different geographical reference. Typically, it is $30-35 \%$ of all primary glomerular diseases in Asia, but can be up to $45 \%$. It accounts for $20 \%$ of all biopsies in Europe and $10 \%$ of all biopsies in North America. This wide variation in incidence is partly attributable to differing indication for kidney biopsy in Asia, compared with those in North America. Recently in the USA IgAN was also reported to be the most common primary glomerulopathy in young adult Caucasians.

$\operatorname{IgA}$ nephropathy is an example of $\operatorname{Ig} \mathrm{A}_{1}$ immune complex disease. ). Poorly galactosylated $\mathrm{IgA}_{1} \mathrm{O}$ glyco-forms form high molecular weight circulating immune complexes, either through self-aggregation or through the generation or IgG and IgA hinge region specific autoantibodies $(4,5,6)$.Episodic gross haematuria is the presenting complaint in $40-50 \%$ patients usually following upper respiratory infection (synpharyngitis or syninfective)[7]. 
Rapid progression to ESKD is an uncommon feature with IgAN in various studies. In our patient, the rapid clinical deterioration and histopathological presence of cellular/fibrocellular crescent, fibrinoid necrosis along with global sclerosis were suggestive of active glomerular injury on the background of chronic kidney damage, although patient never complained any chronic symptoms of duration of 3 months or more. Such a co-incidence of chronic disease with crescentic GN in IgAN makes this case worth reporting.

In a study by Huang et al, 14 patients with IgAN and serological positive ANCA were reviewed.[8]

Their conclusion was that patients with IgAN and ANCAs positivity commonly show clinical features of rapidly deteriorating kidney disease. ${ }^{[8]}$

In a study from $\mathrm{Lv}$ et al,[9,10] 113 Chinese patients with crescentic IgAN were recruited from various centres. They found that presence of crescent carried a poor prognosis, and nearly $70 \%$ of them progressed to ESRD in 5 years. The initial serum creatinine ( $\mathrm{Sr}$. Cr) concentration was the strongest risk factor for kidney failure. Patients with lower creatinine at presentation ( $\mathrm{Sr} . \mathrm{Cr}<2.7 \mathrm{mg} / \mathrm{dL}$ ) had a good prognosis. Patients with higher $(\mathrm{Sr} . \mathrm{Cr}>6.8 \mathrm{mg} / \mathrm{dL}$ ) had lesser recovery and were dialysis dependent, even after aggressive immunosuppressive therapy.

This case illustrates one of a small percentage of patients with IgAN that can develop a rapidly progressive form of the disease leading to ESRD.

Some clinical and laboratory findings at the time of diagnosis can potentially help stratify the severity of disease.[11,12,13]

Elevated serum creatinine concentration, hypertension, persistent proteinuria follows a worse prognosis. In a study from Japan on 2, 270 patients with IgAN, there was a strong correlation with cumulative incidence of ESRD at 7 years with the $\mathrm{Sr}$. $\mathrm{Cr}$ at presentation ( $\mathrm{P}$ value for the trend $<0.001$ ).[11]

Histological findings on renal biopsy in patients with IgAN studied on the basis of MEST (Oxford histologic classification system) has improved the ability to identify patients with a poor renal prognosis.[14]

A combined prediction score that estimates the 5-year risk of developing ESRD known as the absolute renal risk score was developed in a Japanese cohort of 698 untreated patients and then validated in a separate cohort of 702 patients.[15]

Points were assigned according to two clinical risk factors1. 24-h urine protein excretion

2. eGFR)

and three of the four Oxford classification criteria.

In a study of 5 children with RPGN by Welch et al. All had hypertension and 3 had nephrotic syndrome. Hypertension, renal insufficiency and nephrotic syndrome were consistent marker of rapid decline in IgAN patient and they were resistant to therapy with rapid decline of renal function. These three features were absent in comparative uncomplicated IgAN cases. ${ }^{[16]}$

\section{Conclusion and some Unresolved Issues-}

My patient was a case of IgA nephropathy, RPRF course, extensive crescents with $>50 \%$ global sclerosis. Her initial infective episode was well managed with antibiotics and stopping immunosuppressant. Antifungal was not given because she improved with antibiotics. She was dialysis dependent because of obvious tissue diagnosis- DGGS and superimposed crescents of chronic type. She ultimately succumbed due to second infective episode.

Some Unresolved issues associated with the Patient were-

1. She never complained of haematuria which is a predominant clinical manifestation of IgAN.

2. Tissue diagnosis suggesting global sclerosis of $>50 \%$ glomeruli and superimposed crescents of chronic type is not compatible with such an acute and fulminant clinical course of IgAN.

\section{FINAL DIAGNOSIS}

RPGN, IgA Nephropathy with superimposed global crescents as well as crescents in the remaining glomeruli with varying stages. Anaemia, HTN, Dialysis Dependent. No Comorbidity.

\section{REFERENCES}

[1] Donadio JV, Grande JP. IgA nephropathy. N Engl J Med 2002;347(10):738-48.

[2] Rocatello D, Ferro M, Coppo R, et al. Treatment of rapidly progressive IgA nephropathy. In: Clarkson A, Woodroffe A, eds. IgA Nephropathy: pathogenesis and treatment. Vol. 111. Basel: Karger 1995: p. 177-1483.

[3] Wyatt RJ, Julian BA. IgA nephropathy. N Engl J Med 2013;368(25):2402-14.

[4] Barrat J, Feehally J. Primary IgA nephropathy: new insights into pathogenesis. Semin Nephrol 2011;31(4):349-60.

[5] Barratt J, Smith AC, Feehally J. The pathogenic role of IgA1 O-linked glycosylation in the pathogenesis of IgA nephropathy (review article), Nephrology (Carlton) 2007;12(3):275-84.

[6] Barratt J, Smith AC, Molyneux K, et al. Immunopathogenesis of IgAN. Semin Immunopathol 2007;29(4):427-43.

[7] Barratt J, Feehally J. IgA nephropathy and related disorder. NKF Primer on kidney diseases. 2014: p. 18592.

[8] Huang X, Wang Y, Xie L, et al. IgA nephropathy with anti-neutrophil cytoplasmic antibody seropositivity. Clin Nephrol 2015;84(3):156-64.

[9] Rojas-Rivera J, Fernandez-Juarez G, Praga M. Rapidly progressive IgA nephropathy: a form of vasculitis or a complement-mediated disease? Clin Kidney J 2015;8(5):477-81.

[10] Lv J, Yang Y, Zhang H, et al. Prediction of outcomes in crescentic IgA nephropathy in a multicenter cohort study. J Am Soc Nephrol 2013;24(12):2118-25.

[11] Wakai K, Kawamura T, Endoh M, et al. A scoring system to predict renal outcome in IgA nephropathy: from a nationwide prospective study. Nephrol Dial Transplant 2006;21(10):2800-8.

[12] Berthoux F, Mohey H, Laurent B, et al. Predicting the risk for dialysis or death in IgA nephropathy. J Am Soc Nephrol 2011;22(4):752-61.

[13] Strippoli GF, Manno C, Schena FP. An evidence based survey of therapeutic options for IgA nephropathy: assessment and criticism. AM J Kidney Dis 2003;41(6):1129-39. 
[14] Coppo R, Troyanov S, Camilla R, et al. The Oxford IgA nephropathy clinicopathological classification is valid for children as well as adults. Kidney Int 2010;77(10):921-7.

[15] Tanaka S, Ninomiya T, Katafuchi R, et al. Development $\&$ validation of a prediction rule using the Oxford classification in IgA nephropathy. Clin J Am Soc Nephrol 2013;8(12):2082-90.
[16] Welch TR, McAdams AJ, Berry A. Rapidly progressive IgA nephropathy. Am J Dis Child 1988;142(7):789-93. 